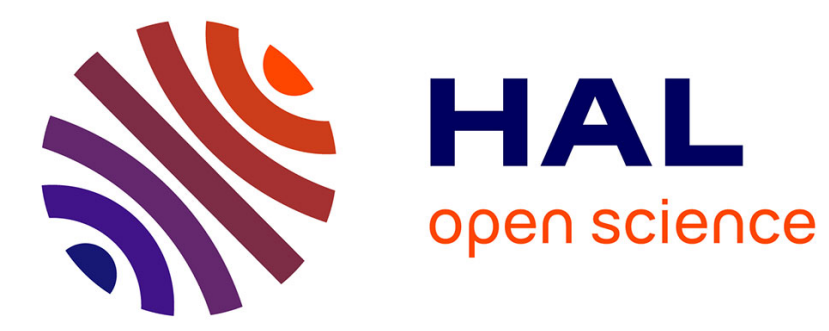

\title{
Optical phase modulation by natural eye movements: application to time-domain FF-OCT image retrieval
}

Viacheslav Mazlin, Peng Xiao, Kristina Irsch, Jules Scholler, Kassandra

Groux, Kate Grieve, Mathias Fink, A. Claude Boccara

\section{To cite this version:}

Viacheslav Mazlin, Peng Xiao, Kristina Irsch, Jules Scholler, Kassandra Groux, et al.. Optical phase modulation by natural eye movements: application to time-domain FF-OCT image retrieval. Biomedical optics express, 2022, 13 (2), pp.902. 10.1364/boe.445393 . hal-03566130

\section{HAL Id: hal-03566130 \\ https://hal.sorbonne-universite.fr/hal-03566130}

Submitted on 11 Feb 2022

HAL is a multi-disciplinary open access archive for the deposit and dissemination of scientific research documents, whether they are published or not. The documents may come from teaching and research institutions in France or abroad, or from public or private research centers.
L'archive ouverte pluridisciplinaire HAL, est destinée au dépôt et à la diffusion de documents scientifiques de niveau recherche, publiés ou non, émanant des établissements d'enseignement et de recherche français ou étrangers, des laboratoires publics ou privés. 


\title{
Optical phase modulation by natural eye movements: application to time-domain FF-OCT image retrieval
}

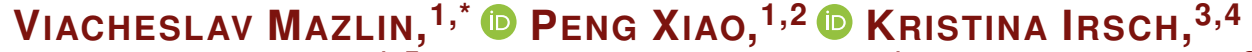 \\ Jules SCHOLler, ${ }^{1,5}$ (1) KASSANDra GrouX, ${ }^{1}$ (i) KATE Grieve, ${ }^{3,4}$ \\ MATHIAS FINK, ${ }^{1}$ AND A. ClaUde BOCCARA ${ }^{1}$ \\ ${ }^{1}$ ESPCI Paris, PSL University, CNRS, Langevin Institute, 1 Rue Jussieu, 75005 Paris, France \\ ${ }^{2}$ State Key Laboratory of Ophthalmology, Zhongshan Ophthalmic Center, Sun Yat-sen University, 510060, \\ Guangzhou, China \\ ${ }^{3}$ Vision Institute, Sorbonne University, CNRS, INSERM, 17 Rue Moreau, 75012 Paris, France \\ ${ }^{4}$ Quinze-Vingts National Ophthalmology Hospital, 28 Rue de Charenton, 75012 Paris, France \\ ${ }^{5}$ Wyss Center for Bio and Neuroengineering, Chem. des Mines 9, 1202 Geneva, Switzerland \\ *mazlin.slava@gmail.com
}

\begin{abstract}
Eye movements are commonly seen as an obstacle to high-resolution ophthalmic imaging. In this context we study the natural axial movements of the in vivo human eye and show that they can be used to modulate the optical phase and retrieve tomographic images via time-domain full-field optical coherence tomography (TD-FF-OCT). This approach opens a path to a simplified ophthalmic TD-FF-OCT device, operating without the usual piezo motor-camera synchronization. The device demonstrates in vivo human corneal images under the different image retrieval schemes (2-phase and 4-phase) and different exposure times $(3.5 \mathrm{~ms}, 10 \mathrm{~ms}$, $20 \mathrm{~ms}$ ). Data on eye movements, acquired with a spectral-domain OCT with axial eye tracking (180 B-scans/s), are used to study the influence of ocular motion on the probability of capturing high-signal tomographic images without phase washout. The optimal combinations of camera acquisition speed and amplitude of piezo modulation are proposed and discussed.
\end{abstract}

(C) 2022 Optica Publishing Group under the terms of the Optica Open Access Publishing Agreement

\section{Introduction}

The human eye is constantly moving. Many of the movements are caused by unconscious physiological processes such as heartbeat, breathing and brain-induced saccades [1,2], and therefore cannot be completely suppressed. Ophthalmic imaging instruments produce an image by collecting light over a certain amount of time (e.g. integration time of the detector). When this time spans several milliseconds, the eye cannot be considered static as the movements affect the image quality.

Optical coherence tomography (OCT) $[3,4]$ is the clinical gold standard method for crosssectional imaging of the eye. The principle of OCT is based on broadband light interferometry, which allows one to discriminate different layers of the imaged sample. Interferometry is susceptible to sample motion, in particular motion manifesting itself in the axial direction, which can induce optical phase washout and phase wrapping phenomena [5,6]. In this article we will refer to phase washout as a process of tomographic signal fading due to additional optical phase shift, induced by the axial sample motion during the acquisition of the elementary tomographic data. In the example of conventional spectral-domain OCT, the tomographic signal completely fades if the axial movement of the sample produces a $\pi$ optical phase shift (corresponding to a movement of a few hundred nanometers) during the acquisition of a 1D axial reflectivity profile (A-line) of the sample. Phase wrapping occurs when the sample motion produces a large phase-shift lying outside of the $[-\pi, \pi]$ interval. The latter does not necessarily result in signal 
loss, but creates ambiguities in the measurements. The degree to which the tomographic signal is affected by the movements depends on the particular type of OCT.

Today, spectral-domain OCT (SD-OCT) is the most commonly used type of OCT. SD-OCT reconstructs 2D cross-sectional image (B-scan) by stacking scanned A-lines, with each A-line being acquired using a line-scan camera embedded into a spectrometer. Line-scan cameras have a typical integration time of a few microseconds - sufficiently short to avoid phase washout during the A-line capture. If necessary, the residual A-line artifacts can be completely suppressed by using pulsed light, that effectively shortens the exposure time of the camera [7]. Motion artifacts are however more pronounced in the final B-scan image, as it is formed by point-by-point laser beam scanning in the en face plane. These artifacts have become much less visible in recent years given the remarkable progress in increasing the imaging speed, today reaching over 250 000 A-scans/s $[8,9]$.

Swept-source OCT (SS-OCT) $[10,11]$ is another modality which acquires spectral data like SD-OCT, but employs a tunable wavelength laser source (swept-source) and a photodetector instead of spectrometer. Although the photodetector needs to acquire data sequentially over a period of time, this period can be very short thanks to the fast A/D acquisition electronics operating at $\mathrm{GHz}$ speeds. It takes less than a $\mu$ s to capture the entire spectrum, which diminishes the phase washout effect [7,12]. Moreover, the A-line rate can be increased to several $\mathrm{MHz}$ $[13,14]$, which together with the motion correction software led to further suppressed motion artefacts in the final B-scan and volumetric images.

While the above OCT methods have a remarkably fast imaging speed along the axial direction $(\sim \mu \mathrm{s})$, imaging in the lateral direction takes a longer time $(\sim \mathrm{ms})$, as it typically requires a point-by-point laser beam scanning in a $2 \mathrm{D}$ plane with a galvanometer mirror system. In an alternative to point-scanning, a new class of parallel acquisition methods were proposed, including line-field SD-OCT (LF-SD-OCT) [15] and Fourier-domain full-field OCT (FD-FF-OCT) (also known as FF-SS-OCT) [16]. These instruments increased dimensionality of data acquisition by substituting the $0 \mathrm{D}$ photodetectors and $1 \mathrm{D}$ line-scan cameras with 2D cameras. This enabled increase of the effective A-line rate up to $\sim 2 \mathrm{MHz}$ for LF-SD-OCT $[17,18]$ and $\sim 40 \mathrm{MHz}$ for FD-FF-OCT [19-21]. Similar to SS-OCT, FD-FF-OCT needs to acquire spectral information over time. It takes typically $\sim 10 \mathrm{~ms}$ to capture 500 images at a 60,000 images/s frame rate and retrieve the entire spectrum. This is a relatively long time $(\sim 1,000$ times longer than the SS-OCT exposure time), during which the eye can potentially shift by more than several hundred nanometers, leading to $\pi$ phase shift and phase washout. In practice, the washout effect is less pronounced, potentially because the spectral information is captured sequentially in small spectral samples, each of them being acquired in a fraction of the total exposure time $(\sim 10 / 500=20 \mu \mathrm{s})$ [12]. The remaining eye movement artifacts can be suppressed via numerical motion correction in post-processing [22]. LF-SD-OCT acquires the spectrum in a single camera shot, like SD-OCT, which in principle makes it less sensitive to phase washout, assuming the camera exposure time is sufficiently short.

Another type of parallel OCT is time-domain full-field OCT (TD-FF-OCT). Contrary to the spectral-domain OCT methods, which obtain the best results with static interferometer arms (i.e. no optical phase shift), TD-FF-OCT by design requires phase shifting between the arms. This can be achieved by modulating the optical path length of the reference arm with a piezo-motor [23], by using the two physically separated synchronized cameras [24,25], by tilting the reference beam axis in a holographic approach [26], or by using the metabolic cell dynamics [27,28], etc. Recent developments in fast and high-full-well-capacity (FWC) 2D cameras enabled eye imaging applications of TD-FF-OCT, such as $600 \mathrm{MHz}$ (en face pixels/s) angiography in the anterior human eye with capability of tracking individual blood cells [29], along with corneal [29-31] and retinal $[32,33]$ imaging. In most cases the tomographic images were reconstructed from 2 phase-modulated (with piezo-motor) camera frames captured in $\sim 4 \mathrm{~ms}$. 
In this article, we study the effect that the eye movements play during the acquisition of tomographic images in TD-FF-OCT. We show that the natural ocular movements can (with high probability) shift the eye of the fixated subject axially by many hundreds of nanometers during one millisecond. This suggests that the eye movements themselves can be used for modulation of the optical phase, substituting the piezo-modulation. In this way, eye movements are seen not as an obstacle, contrary to the methods above, but become an essential part of the image reconstruction process. We show that the benefit expected from removing the piezo-modulation depends on the exposure time of the optical system. At exposure times of about $1 \mathrm{~ms}$ and above, the ocular movements alone provide sufficient phase shifting. The signal-to-noise ratio (SNR) is slightly reduced compared to the piezo-assisted shifting $\left(\mathrm{SNR}_{\text {piezo }} / \mathrm{SNR}_{\text {no piezo }}=1.3\right)$, however the mismatch between the two decreases at the longer exposure times. At shorter exposures below $1 \mathrm{~ms}$ the eye movements are too slow to provide a sufficient phase shift and the piezo modulation becomes a requirement.

\section{Materials and methods}

\subsection{SD-OCT with axial eye tracking}

We measured the axial movements of the eye (in human cornea in vivo) using a $100 \mathrm{kHz}$ SD-OCT device, resembling that in article [29]. In brief, the SD-OCT is based on a commercial GAN510 (Thorlabs, USA) (Fig. 1).

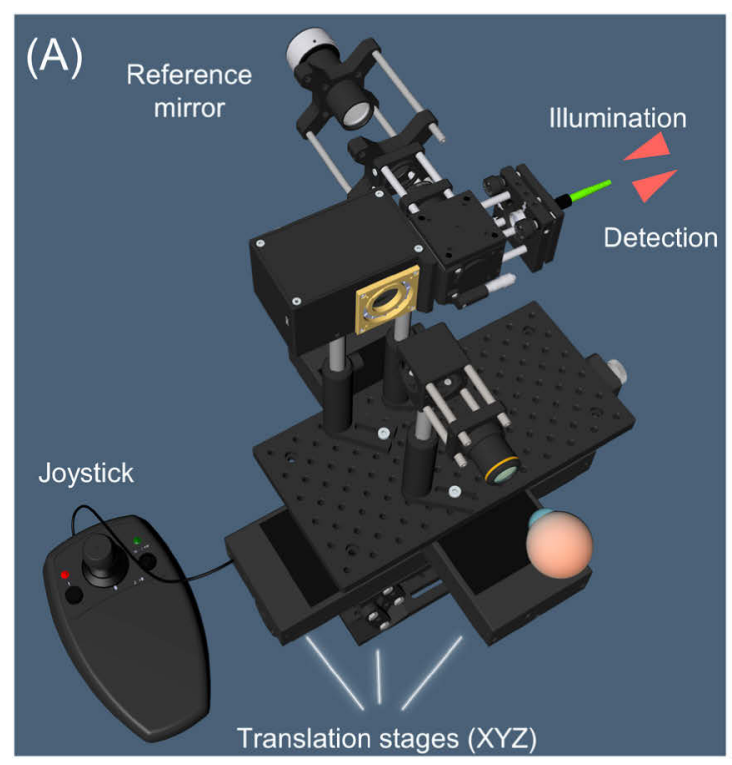

(B)

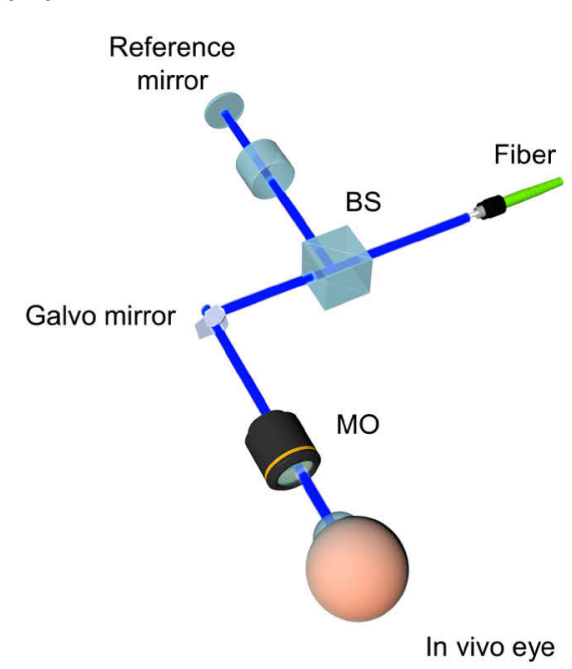

Fig. 1. Detailed (A) and schematic (B) illustrations of SD-OCT, used to measure the axial eye movements. BS: beamsplitter. MO: microscope objective. Spectrometer is not shown. Interferometer is mounted on $\mathrm{XYZ}$ translation stages, useful for aligning with respect to the eye.

It consists of the interferometer with 10× microscope objectives (LMPLN10XIR, Olympus), detecting spectrometer, galvanometric mirror system (OCTP-900(/M), Thorlabs, USA) and superluminescent diode (SLD). The galvanometric mirror rapidly scans a collimated light beam laterally at $100 \mathrm{kHz}$ to form a 2D cross-sectional B-scan image. The SLD has central wavelength of $930 \mathrm{~nm}$ and spectral bandwidth of $40 \mathrm{~nm}$. Dimensions of the B-scan were $1.25 \mathrm{~mm}$ (lateral) 
$2.7 \mathrm{~mm}$ (axial). This corresponded to 512 pixels (lateral) x 1024 pixels (axial), thus 1 lateral pixel $=2.4 \mu \mathrm{m}, 1$ axial pixel $=2.6 \mu \mathrm{m}$.

We performed axial tracking by detecting the position of the reflective peak signal from the air-tear film interface. In this case SD-OCT is used in topography configuration that determines the position of a single non-overlapping peak. The single peak position can be detected with a better resolution than the theoretical $3.9 \mu \mathrm{m}$ (in the cornea), as determined by the Rayleigh criterion (i.e. criterion for discriminating between the two overlapping Gaussian peaks) and the spectral bandwidth. The peak was located via Labview software (National Instruments, USA) by averaging the B-scan along the lateral dimension and applying the Peak detector virtual instrument (National Instruments, USA) with sub-pixel accuracy. Axial movements of the eye were tracked at $180 \mathrm{~Hz}$, locating a new corneal surface position every $5.5 \mathrm{~ms}$, over 20-30 seconds.

\subsection{TD-FF-OCT with enabled and disabled piezo motor}

We used TD-FF-OCT to obtain en face tomographic images of the human cornea. The design of TD-FF-OCT is similar to that reported in [29-31]. In brief, the device is a Linnik interferometer, equipped with the two microscope objectives, a 2D camera and an light-emitting diode (LED) source of low spatial and temporal coherence (Fig. 2).

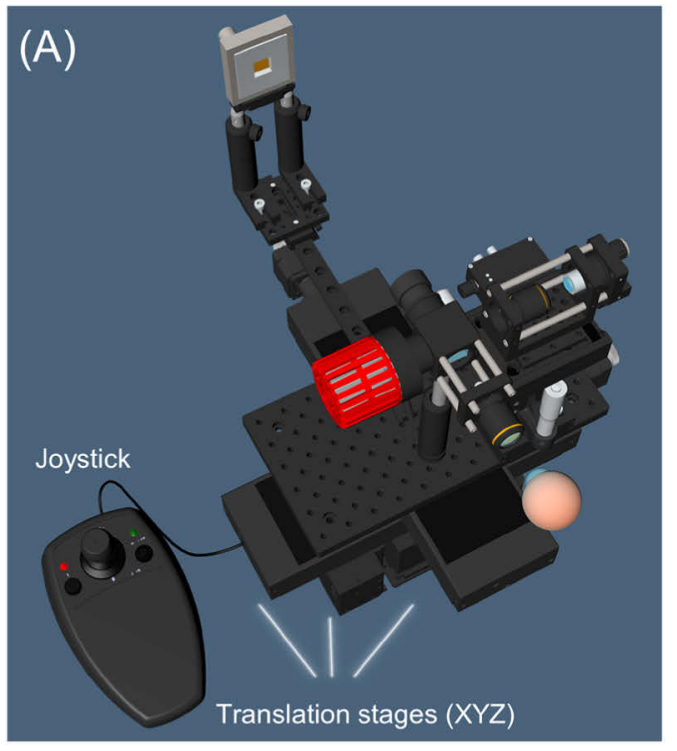

(B)

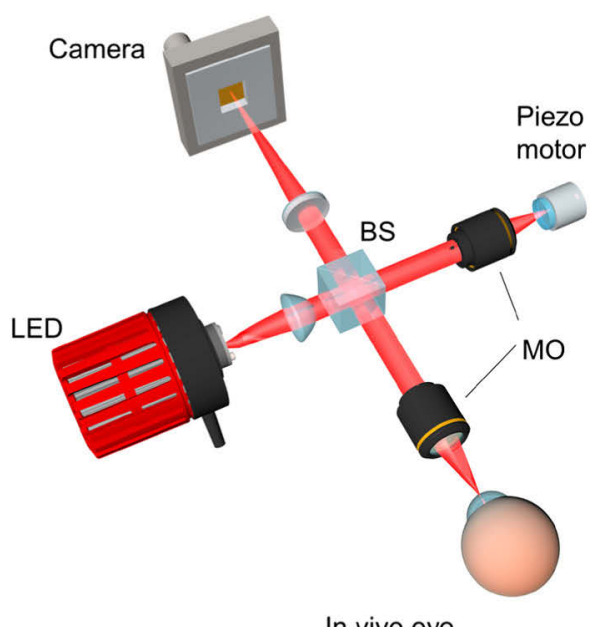

In vivo eye

Fig. 2. Detailed (A) and schematic (B) illustrations of TD-FF-OCT, used to image the in vivo human cornea. BS: 50:50 beamsplitter. MO: microscope objectives. The interferometer is mounted on $\mathrm{XYZ}$ translation stages, useful for aligning with respect to the eye. The piezo motor in the reference arm, that holds the mirror, may be disabled, leaving the axial movements of the eye to be responsible for optical phase modulation.

Microscope objectives (LMPLN10XIR, Olympus, Japan) and a $250 \mathrm{~mm}$ tube lens provide a $13 \times$ magnification (1.1 mm field of view), $18 \mathrm{~mm}$ working distance and $1.7 \mu \mathrm{m}$ lateral resolution. The LED (M850LP1, Thorlabs, USA) has a central wavelength of $850 \mathrm{~nm}$ and a spectral bandwidth of $30 \mathrm{~nm}$, resulting in an axial resolution of $7.7 \mu \mathrm{m}$ (in the cornea). The sensor is a $1440 \times 1440$ pixels CMOS camera with a high $2 \mathrm{Me}^{-}$full well capacity (Q-2A750-CXP, Adimec, Netherlands). Camera imaging speed was 550 frames per second (fps) (1.75 ms camera exposure time). An absorptive neutral density (ND) filter of 4\% reflectivity (NENIR550B, Thorlabs, USA) was used as a reference mirror to achieve high detection sensitivity and avoid multiple 
reflections. The filter was mounted on a piezo motor (STr-25/150/6, Piezomechanik GmbH, Germany), synchronized with the camera, to capture several phase-shifted consecutive images. Modulation amplitude of the piezo motor could be adjusted or disabled to perform phase-shifting only with the axial movements of the eye. The interferometer was mounted on the three high-load translation stages (two NRT150/M, Thorlabs, USA and MLJ150/M, Thorlabs, USA) enabling positioning in three axes.

\subsection{In vivo imaging}

Approval for the study was obtained (study number 2019-A00942-55), in conformity with French regulations, from the CPP (Comité de Protection de Personnes) Sud-Est III de Bron and ANSM (Agence Nationale de Sécurité du Médicament et des Produits de Santé). The study was carried out on two healthy subjects, which was confirmed with eye examinations at the Quinze Vingts National Ophthalmology hospital preceding the experiment. Prior to experimental procedures, which adhered to the tenets of the Declaration of Helsinki, informed consent was obtained from the subjects after the nature of the study was explained. The subjects were asked to rest their chin and temples on a custom-made headrest with three fixation points ( 1 for the chin and 2 for the left and right sides of the forehead) and look at a fixation target within the device. Examination was non-contact and did not involve instillation of eye drops into the eye. The SD-OCT used a scanning light beam and the TD-FF-OCT used a pulsating light to keep the light exposure significantly below the maximum permissible level, as detailed in [29].

\section{Results}

\subsection{Analysis of axial eye movements}

Axial eye movements were measured using a fast SD-OCT with corneal tracking (Fig. 1). A new corneal position was recorded every $5.5 \mathrm{~ms}$ over a duration of 30 seconds (Fig. 3). Such a long period enables exploration of a wide diversity of eye movements. The amplitude of movements ranged from $-150 \mu \mathrm{m}$ to $150 \mu \mathrm{m}$. The main contributors to the motion were fast heartbeat around $1 \mathrm{~Hz}$ with higher harmonics at $2 \mathrm{~Hz}, 3 \mathrm{~Hz}$, etc. and slow breathing at $0.34 \mathrm{~Hz}$, in agreement with the literature [1].

Our primary goal is to study the movements on a shorter (millisecond) time scale. On that scale eye position changes smoothly with time due to limited frequency of physiological movements (see zoom in the rapidly changing part of Fig. 3(A)). This allows a linear interpolation of the experimental data with a finer time step than the experimentally recorded $5.5 \mathrm{~ms}$ and a finer axial accuracy. In order to analyze the influence of eye movements, we divided the entire timeline into sequences of virtual camera frames (with exposure times of $0.1 \mathrm{~ms}, 1 \mathrm{~ms}$, etc.) and checked how the corneal position and optical phase change from one virtual frame to another. Figure 4 shows the distributions of all possible axial corneal shifts, optical phase shifts and velocities happening between the different consecutive frames over the entire 30 seconds timeline. As expected, the shorter the time of the frame (exposure time), the smaller is the eye displacement happening during this time, and therefore, the smaller is the influence of the movements on the acquired images. During the exposure times below $0.1 \mathrm{~ms}$ the eye shifts by less than $100 \mathrm{~nm}$. This increases the optical path length of light by $200 \mathrm{~nm}$ (taking into account the double optical pass to the sample and back), equivalent to an optical phase shift of $\pi / 2$. Here we considered a light wavelength of $850 \mathrm{~nm}$, commonly used in ophthalmology. This highlights that instruments with even shorter (microsecond) exposure times (SD-OCT, SS-OCT, LF-SD-OCT) are almost insensitive to fringe washout ( $\pi$ phase shift). On the other hand, during exposure times longer than $1 \mathrm{~ms}$ the eye can shift by $1 \mu \mathrm{m}$ and more, leading to large optical phase shifts of $\pi$ and more. It is insteresting to note that while such large movements can potentially lead to phase washout in spectral-domain methods, time-domain techniques (e.g. TD-FF-OCT) can potentially use them 
(A)

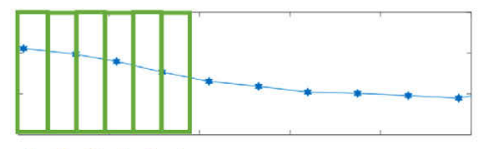

$123456 \cdots$

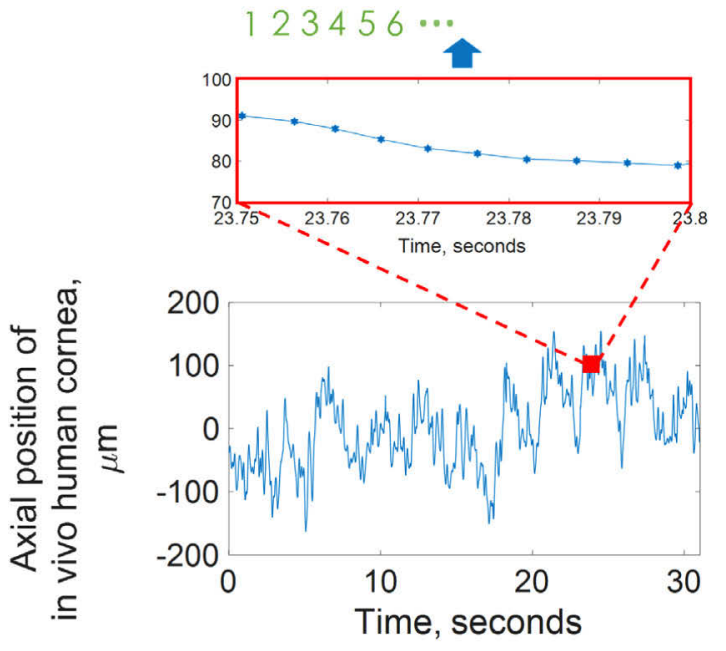

(B)

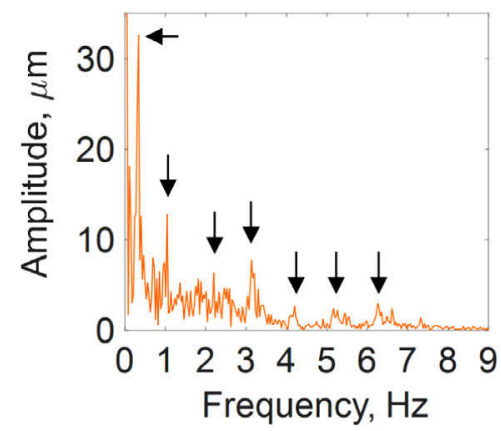

Fig. 3. (A) Axial movements of in vivo human cornea, measured with SD-OCT. The source data underlying the plot are provided in Dataset 1, Ref. [34]. The long period of recording enables exploration of wide diversity of eye movements. Red square (zoomed image) highlights the linearity of eye movements on a ms time scale. The entire plot can be divided into the imaginary time windows (e.g. $1 \mathrm{~ms}, 3.5 \mathrm{~ms}, 10 \mathrm{~ms}$ etc.), shown in green. (B) Fourier transform of the main plot (A). Peaks corresponding to breathing at $0.34 \mathrm{~Hz}$ and heartbeat at $1 \mathrm{~Hz}, 2 \mathrm{~Hz}, 3 \mathrm{~Hz}$ are visible in agreement with the literature [1].

to their advantage for optical phase modulation. Figure 4(D) highlights that large phase shifts (> $\pi / 2$ ) account for almost $80 \%$ of all shifts within $1 \mathrm{~ms}$, for almost $100 \%$ within $10 \mathrm{~ms}$ and for only $3 \%$ within $0.1 \mathrm{~ms}$ and below.

\subsection{Analysis of phase modulation with eye movements in TD-FF-OCT}

Below we will analyze the influence of eye movements on TD-FF-OCT signal. The simplest TD-FF-OCT image retrieval scheme requires two consecutive camera frames to reconstruct a tomographic image [35]. Below, by the exposure time we will mean the time required to capture the tomographic TD-FF-OCT image (equal to the time of 2 camera frames). The tomographic signal is the highest when the optical phase shift (supposed here to be instantaneous) between those frames equals $\pi, 3 \pi$, etc. and is absent at $0,2 \pi, 4 \pi$, etc. The higher order phase shifts of $5 \pi, 7 \pi$ etc. can also produce the highest tomographic signal, as long as the axial corneal shift stays within the coherence length. The latter is typically true for ophthalmic TD-FF-OCT [30], given that during its exposure time of $3.5 \mathrm{~ms}$ ( 2 camera frames, each frame $1.75 \mathrm{~ms}$ ), the axial corneal shifts are still within its coherence gate thickness (axial resolution) of $7.7 \mu \mathrm{m}$ (Fig. 4(A)).

The intensity on the camera can be written generally, as:

$$
I=\frac{I_{0}}{4} \cdot\left\{R_{\text {inc }}+R_{\text {coh-sam }}+R_{\text {ref }}+2 \cdot \sqrt{R_{\text {coh-sam }} \cdot R_{\text {ref }}} \cdot \int_{T_{0}}^{T_{1}} \cos (\varphi+\phi(t)+\psi(t)) d t\right\},
$$

where $I_{0}$ is the photon flux of illumination, $R_{\text {inc }}$ is the 'incoherent' reflectivity from all the sample structures and optical system parts outside of the coherence volume, $R_{\text {coh-sam }}$ is the 'coherent' 


\section{Biomedical Optics EXPRESS}
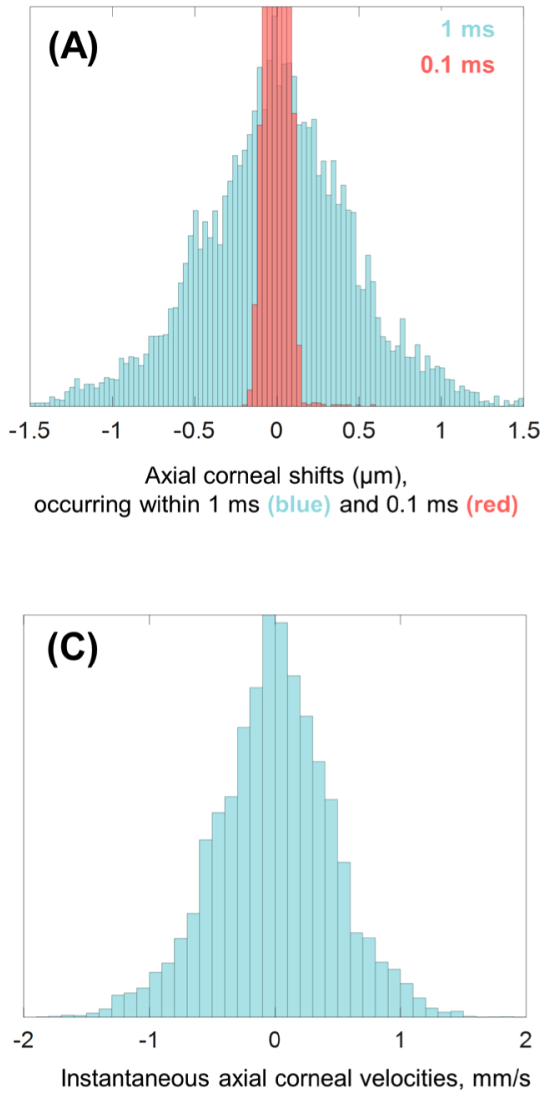
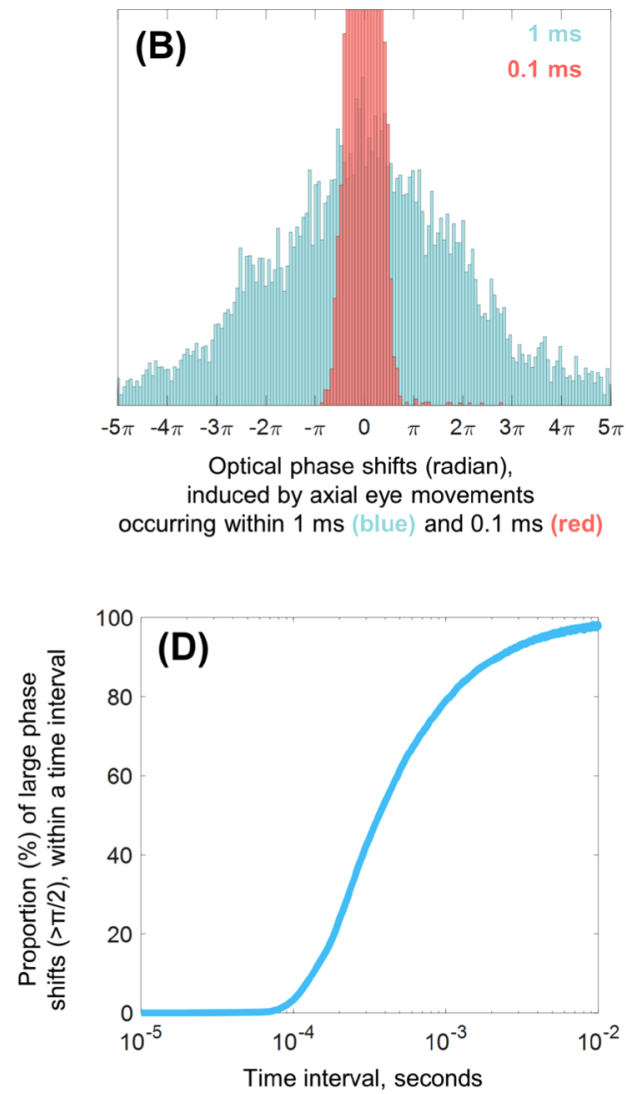

Fig. 4. (A) Distributions of axial corneal position shifts during different time intervals. Data is obtained from Fig. 3(A), divided into imaginary time frames. (B) Distribution of optical phase shifts calculated from (A), assuming an $850 \mathrm{~nm}$ light wavelength. (C) Distribution of instantaneous axial corneal velocities. (D) Proportion of a large phase shift $(>\pi / 2)$ among all shifts happening during different time intervals. The graph is calculated from (B). It should be noted that large phase shifts $(>2 \pi)$ also can produce a high tomographic signal, but for some OCT methods this may require additional phase unwrapping.

reflectivity from all sample structures lying within the coherence volume, $R_{\text {ref }}$ is the reflectivity from the reference mirror, $\varphi$ is the phase difference between the sample and reference signals (denoting fringes in the 2D image), $\phi(t)$ is the additional phase, modulated with the step function in time by the piezo motor, $\psi(t)$ is the additional phase, modulated in time by the movements of the eye and $T_{1}-T_{0}$ is the camera exposure time. Note that the locations of the pixel in the 2D image $(x, y)$ are omitted from the arguments.

For simplicity we consider one pixel $(x, y)$ on the camera, for which the spatial phase $\varphi(x, y)=0$. In order to remove the incoherent part and retrieve the interference amplitude, two images are subtracted and the modulus is taken:

$$
\left|I_{1}-I_{2}\right|=\text { const } \cdot\left|\int_{T_{0}}^{T_{1}} \cos (\phi(t)+\psi(t)) d t-\int_{T_{1}}^{T_{2}} \cos (\phi(t)+\psi(t)) d t\right|,
$$

where const $=I_{0}^{2} \cdot R_{\text {coh-sam }} \cdot R_{\text {ref }}$, while $T_{1}-T_{0}$ and $T_{2}-T_{1}$ are the exposure times for the first and second frames respectively. The module term determines the strength of the tomographic 
signal. If the optical phase shift between the consecutive camera frames is small, then the term in the modulus is small and the tomographic signal is low.

For calculations we will consider the typical parameters for in vivo TD-FF-OCT [28]: $850 \mathrm{~nm}$ light wavelength and exposure time of $3.5 \mathrm{~ms}$ ( 2 camera frames, each frame $1.75 \mathrm{~ms}$ ). We will also rely on a common TD-FF-OCT piezo-modulation scheme, for which $\phi(t)$ equals 0 every first frame and $\pi$ every second frame.

The distribution of possible eye motion induced phase shifts $\psi(t)$ was computed using $3.5 \mathrm{~ms}$ exposure time and the axial movements from Fig. 3. By inserting this distribution into Eq. (2), we obtain the distribution of possible TF-FF-OCT signals in the presence of eye movements (Fig. 5). Signal is normalized to 1.

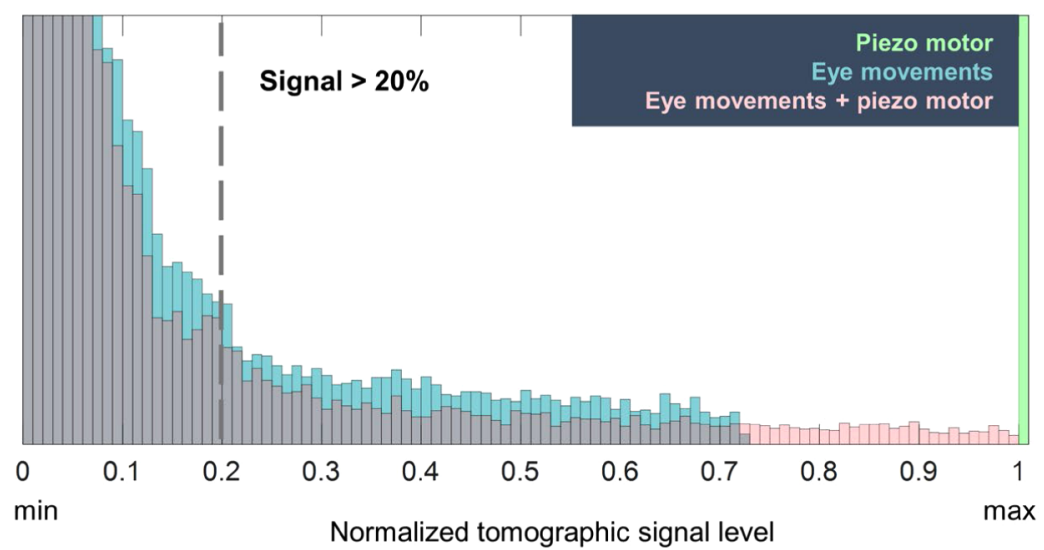

Fig. 5. Distribution of possible TD-FF-OCT signals (normalized) under different sources of phase modulation. The histograms are calculated from Eq. (2) considering the experimental data in Fig. 3, $3.5 \mathrm{~ms}$ exposure time (2 camera frames, each frame $1.75 \mathrm{~ms}$ ) and $850 \mathrm{~nm}$ light wavelength. The signal is the highest (equal to 1), when only the piezo motor contributes to phase modulation in the absence of eye movement. In case of phase modulation with axial eye movements, the tomographic signal can take only values below 0.7 . As discussed below, this results from integration of continuous function of time (phase change with eye movements is a continuous function of time). The addition of discontinuity, such as made with a fast step modulation with a piezo motor, can create tomographic signals of any value. $20 \%$ denotes the arbitrary selected threshold of 'sufficient' tomographic signal that is used for calculating signal statistics.

As expected in case of a static sample and $\pi$ piezo motor modulation between the consecutive frames, TD-FF-OCT signal is always at maximum. A rather unusual distribution of values is obtained if we consider modulation by the in vivo movements of the eye only (without piezo motor). More precisely, if we rely only on the phase-shifting by eye movements, we cannot obtain a TD-FF-OCT signal higher than $70 \%$ of the theoretical maximum. Interestingly, this cannot be explained by the movements of the eye being too small, because, as we have seen above, they frequently give the $\pi, 2 \pi$ and higher phase-shifts between the consecutive frames (for $3.5 \mathrm{~ms}$ exposure time). The reason for such behavior is that the axial eye position (and optical phase) is a continuous function of time. More precisely, in Fig. 3 we saw that on a short ms time scale, axial eye position (and optical phase) is a linear function of time $\psi(t)=a \cdot t$. Then, substituting in Eq. (2) and assuming no piezo modulation, one obtains:

$$
\left|I_{1}-I_{2}\right|=\text { const } \cdot \frac{\left|\sin \left(a T_{1}\right)-\sin \left(a T_{0}\right)-\sin \left(a T_{2}\right)+\sin \left(a T_{1}\right)\right|}{a}
$$


For simplicity we can arbitrarily select the start of the timeline $T_{0}=0, T_{1}=T, T_{2}=2 T$. Then, $a=(\psi(T)-\psi(0)) / T$ and

$$
\left|I_{1}-I_{2}\right|=\text { const } \cdot\left|\frac{\sin (\tau) \cdot(1-\cos (\tau))}{\tau}\right|,
$$

where $\tau=\psi(T)-\psi(0)$. This normalized function can have values only below 0.7 (Fig. 6). This means that TD-FF-OCT signal, modulated by the eye movements (which change linearly on a ms time scale), is limited to $70 \%$ of the theoretical maximum.

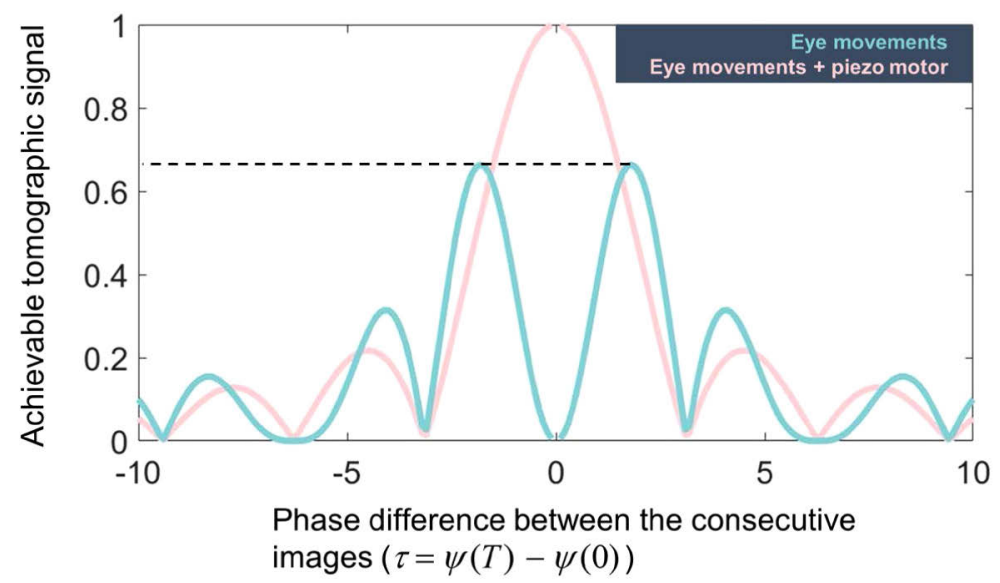

Fig. 6. Values that tomographic signal can take in the cases of modulation with eye movements and piezo motor or with eye movements only.

On the contrary, if in addition to phase shifting with eye movements, we add a step-like discontinuous phase shifting with the piezo motor, Eq. (2) becomes:

$$
\begin{array}{r}
\left|I_{1}-I_{2}\right|=\text { const } \cdot\left|\int_{T_{0}}^{T_{1}} \cos \psi(t) d t-\int_{T_{1}}^{T_{2}} \cos (\pi+\psi(t)) d t\right| \\
\left|I_{1}-I_{2}\right|=\text { const } \cdot\left|\frac{\sin (\tau)}{\tau}\right|
\end{array}
$$

And the function can take any value, which confirms the results in Fig. 5.

It is interesting to note that the lowest tomographic signal is achieved if the eye movements produce $\pi$ phase shift during the acquisition of a single camera frame. This is explained by the fact that as the eye moves continuously and linearly at a millisecond timescale, the camera integrates all the phases between 0 and $\pi$, which leads to phase washout. In the absence of piezo modulation, the highest tomographic signal is reached when the eye movements produce a phase shift close to $\pi / 2$.

As we have seen above, the tomographic signal can take a variety of values depending on the eye movements at each moment. What follows is that the discussion about the deterministic signal should be replaced with a discussion about the signal statistics. Two quantities are useful when evaluating the signal statistics: 1) an average signal level over time and 2) probability of obtaining a signal above a certain threshold level. The latter measure can be used to find the probability of obtaining images with sufficient tomographic signal or equivalently the proportion of images with tomographic signals among all images. The threshold for selecting 'sufficient' 
signal is arbitrary and we set it at $20 \%$ from the theoretical maximal signal. The main conclusions below will remain true for other threshold levels.

Figure 7 shows how these two quantities change depending on the exposure time and phase shift of piezo modulation. The best imaging performance is achieved with the $\pi$ piezo modulation. Nevertheless, depending on the exposure time of the imaging system, the piezo can be removed without sacrificing the signal. When the exposure time is about $0.1 \mathrm{~ms}$, piezo phase shifting is essential - without it only $10 \%$ of images will contain tomographic signal. At the exposure time of $1 \mathrm{~ms}$ and above, the ocular movements start to dominate. The proportion of tomographic images becomes almost independent of the piezo shifting. The SNR advantage at $1 \mathrm{~ms}$ is $\mathrm{SNR}_{\text {piezo }} / \mathrm{SNR}_{\text {no piezo }}=1.3$ and reduces further with the increasing exposure time. As an intermediate conclusion: if one wishes to rely only on the eye movements for phase modulation, it is worth keeping the exposure time at $1 \mathrm{~ms}$ or higher, for which the ocular motion is still sufficiently fast to provide the required phase shift. The additional phase modulation with the piezo motor does not play a significant role at the exposures above $1 \mathrm{~ms}$, whereas at shorter exposures it becomes a requirement because the eye movements are too slow to provide a sufficient phase shift.
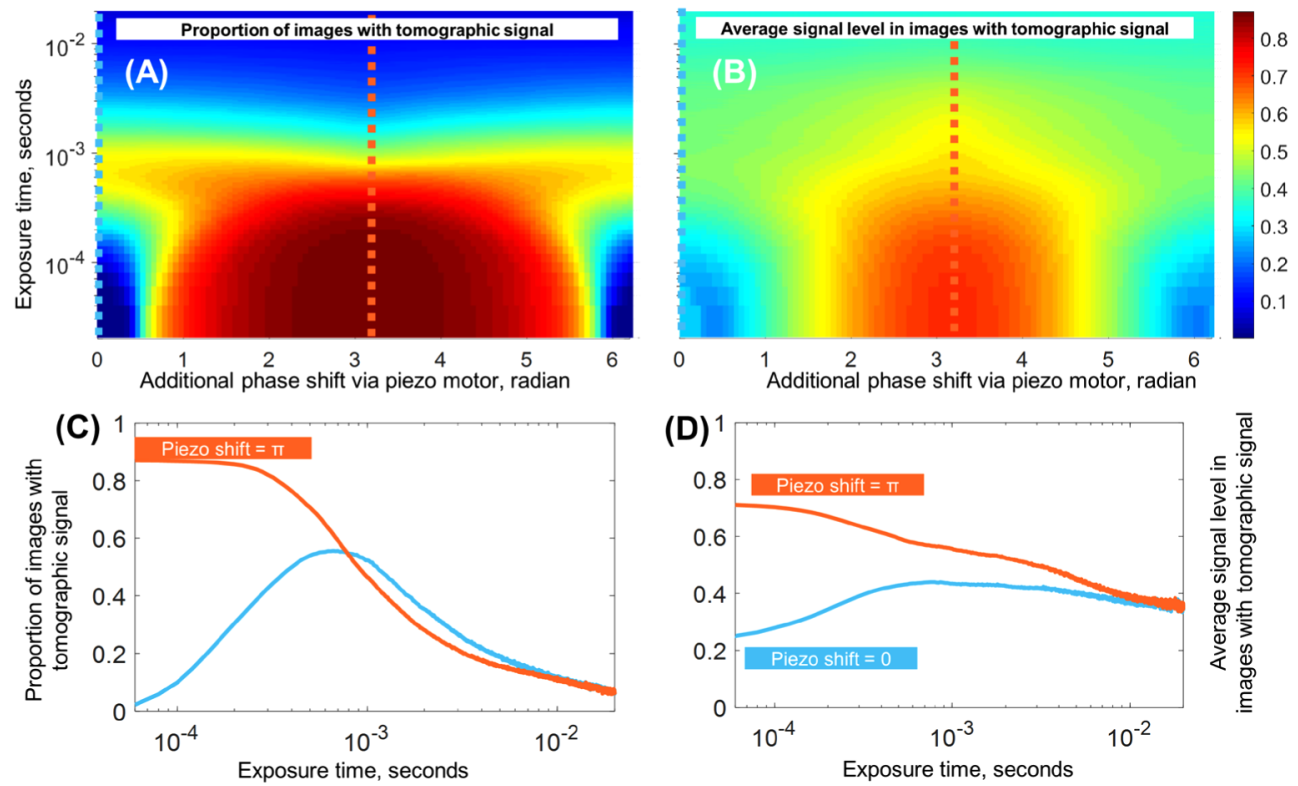

Fig. 7. (A) Proportion of tomographic images in presence of eye movements depending on the exposure time and additional piezo phase shift. By tomographic images we mean images with tomographic signal above $20 \%$ (arbitrarily selected). Here the exposure time is the period required to capture a single tomographic image (reconstructed from the two camera frames). The 2D surface plot is calculated from Eq. (2) with experimental data from Fig. 3(A). (B) Normalized average signal level of tomographic images depending on the exposure time and additional phase shift. (C) Extracted slices from (A). (D) Extracted slices from (B). Color scale is similar for (A) and (B).

Next, we tested the ability of TD-FF-OCT to acquire tomographic images without using a piezo motor. The exposure time (equal to 2 camera frames) was set to $3.5 \mathrm{~ms}$ ( $275 \mathrm{fps}$ ), $10 \mathrm{~ms}$ (100 fps) and $20 \mathrm{~ms}$ (50 fps). With the increasing exposure time, the power of the LED was accordingly reduced to ensure the consistent operation at a level close to saturation of the camera. In all cases we were able to acquire images both with and without piezo motor modulation (Fig. 8). Stromal nuclei were visible in all images. However, in the case of long exposure (20 


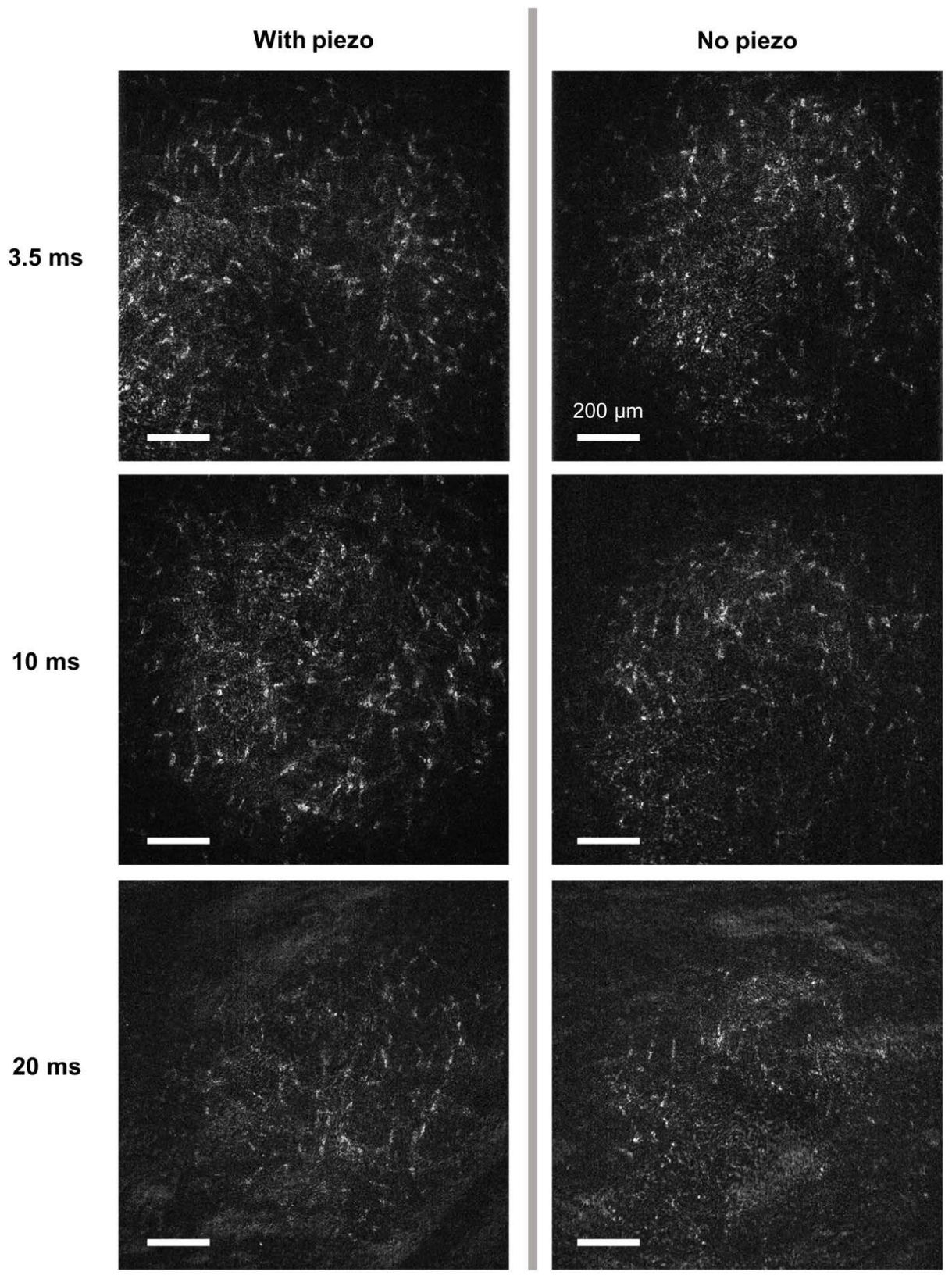

Fig. 8. Comparison of TD-FF-OCT images of human corneal stroma in vivo with and without piezo modulation at different exposure times. Stromal keratocytes are resolved. Exposure time here is the period required to capture two camera frames (single tomographic image). At long exposure time of $20 \mathrm{~ms}$ images contain artefacts related to the defocused view of the corneal surface. All scale bars are $200 \mu \mathrm{m}$. 
ms) the lateral movements created artifacts. More precisely, when the lateral movements are large, the incoherent light outside of the coherence volume is not completely removed during the consecutive image subtraction (see Eq. (1)). Most of the incoherent light originates from the surface reflection from the tear film, therefore in the final image the contrast of the tomographic signal over background is reduced and we see a defocused view of the corneal surface. More details on the influence of motion are provided below in sub-section 3.6.

\subsection{Comparison between healthy subjects}

The experiment and data analysis were repeated on another healthy subject, leading to similar results (Fig. 9). This further highlights the role of common physiological eye movements (such as heartbeat) in fast axial movements and phase modulation.
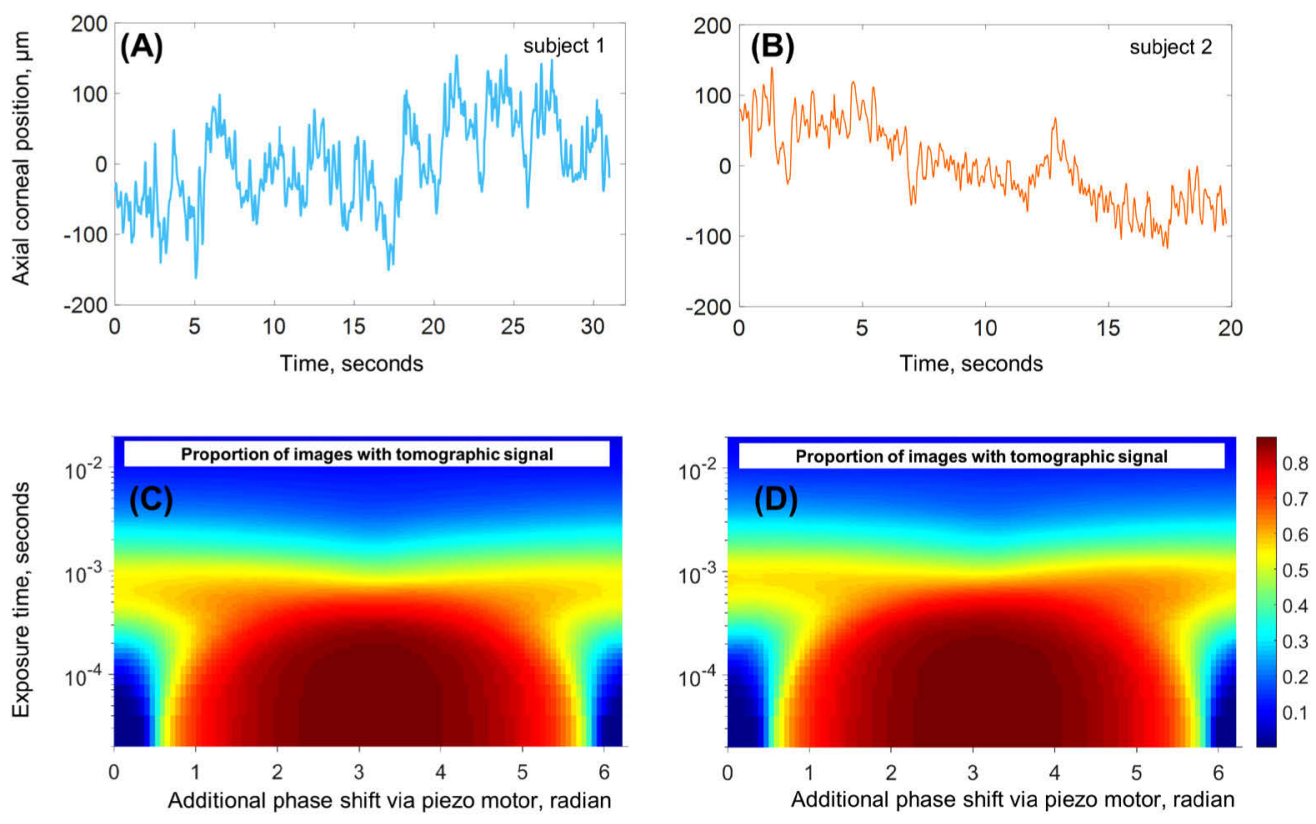

Fig. 9. (A), (B) Comparison of axial movements of in vivo human cornea from two healthy subjects. The source data underlying the plots are provided in Dataset 1, Ref. [34]. (C), (D) Comparison of proportions of tomographic images depending on the exposure time and additional piezo phase shift for two healthy subjects. Color scale is similar for (A) and (B).

\subsection{Reconstruction of TD-FF-OCT signals from multiple images}

Previously we explored the TD-FF-OCT signals reconstructed from only two camera frames. However, thanks to the fast acquisition speed more images are typically available from the same coherence volume. These multiple images can be used to either increase the signal-to-noise ratio (SNR) by averaging or to remove the interference fringe artifacts (4 phase image retrieval scheme). Figures 10 and 11 compare the effectiveness of different image averaging schemes in presence of eye movements and without piezo modulation: averaging of 40 tomographic images, standard deviation of 40 tomographic images and standard deviation of 40 direct camera frames (i.e. frames directly captured by the camera without post-processing). It should be noted that each image is being acquired with a different phase determined by the eye movements. All the schemes reduce the proportion of images having minimal $(0 \%)$ and maximal $(100 \%)$ signals, while increasing the proportion of middle level signals. The schemes differ in the signal level. 
For averaging the majority of images have signal level of $10 \%$, while for standard deviation of tomographic images and direct images this level is $20 \%$ and $30 \%$ respectively. Although the latter method produces large proportion of images with the high tomographic signal, it is not practical as the standard deviation of direct images amplifies not only the signals originating from the coherence volume, but also the motion of irregularities on the ocular surface (Fig. 11). More details on the influence of motion are provided below in sub-section 3.6.

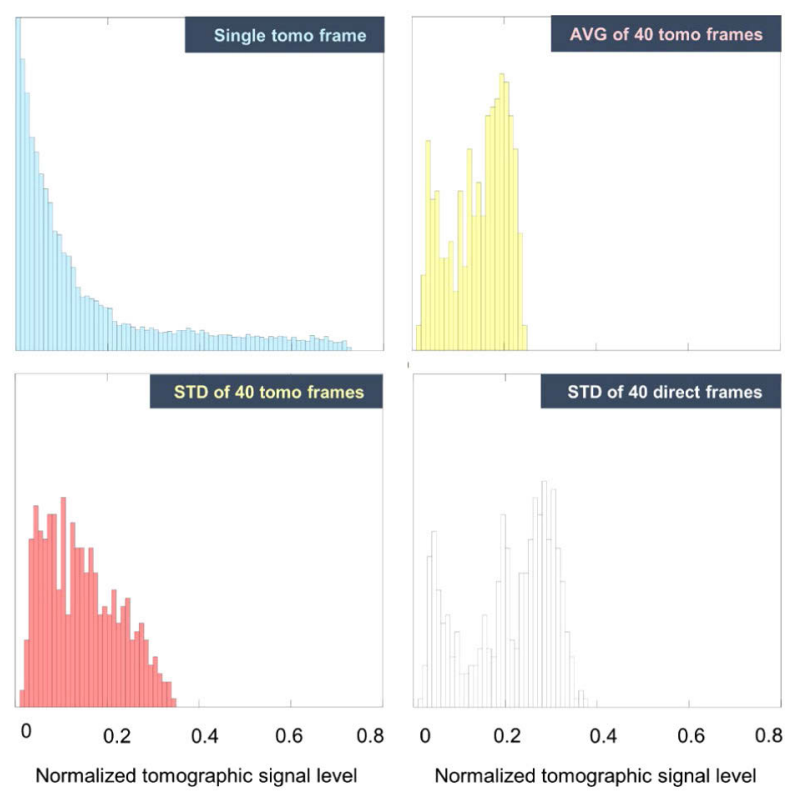

Fig. 10. Distribution of normalized tomographic signal level under different image averaging schemes. The histograms are calculated from Eq. (2) considering the experimental data in Fig. 3, while assuming a $3.5 \mathrm{~ms}$ exposure time, $850 \mathrm{~nm}$ light wavelength and no piezo modulation. Averaging (AVG) or taking the standard deviation (STD) reduces the proportion of images with minimal $(0 \%)$ and maximal $(100 \%)$ signal, while increasing the proportion of mediocre signals.

Another question that is worth asking is: can eye movements be used to acquire tomographic images under a four-phase modulation scheme (in comparison to the two-phase scheme tested before)? The four-phase modulation scheme has an advantage in that it suppresses the pixel phase $\varphi(x, y)$ term (Eq. (1)), which is responsible for the fringe artefacts present in every two-phase tomographic image $[30,31,35]$. While this is of low importance in the irregular tissue interior, the artifacts are particularly visible in uniform reflective layers such as the corneal endothelium. The typical four-phase modulation scheme reconstructs the tomographic image from the four camera frames with phases of: $0, \pi / 2, \pi, 3 \pi / 2$. From the eye motion data (Fig. 3(A)) we calculated the $8 \%$ probability for the sum error in phases of four consecutive images to be below $3 \pi / 4$ (i.e. less than $\pi / 4$ error in each phase shift):

$$
\left|\left(\psi_{1}-\psi_{2}\right)-\pi / 2\right|+\left|\left(\psi_{1}-\psi_{3}\right)-\pi\right|+\left|\left(\psi_{1}-\psi_{4}\right)-3 \pi / 2\right|<3 \pi / 4
$$

Although this probability is low, we were able to acquire four phase images and reveal the endothelial cell mosaic in single TD-FF-OCT frames without the need for frame averaging (Fig. 12). 

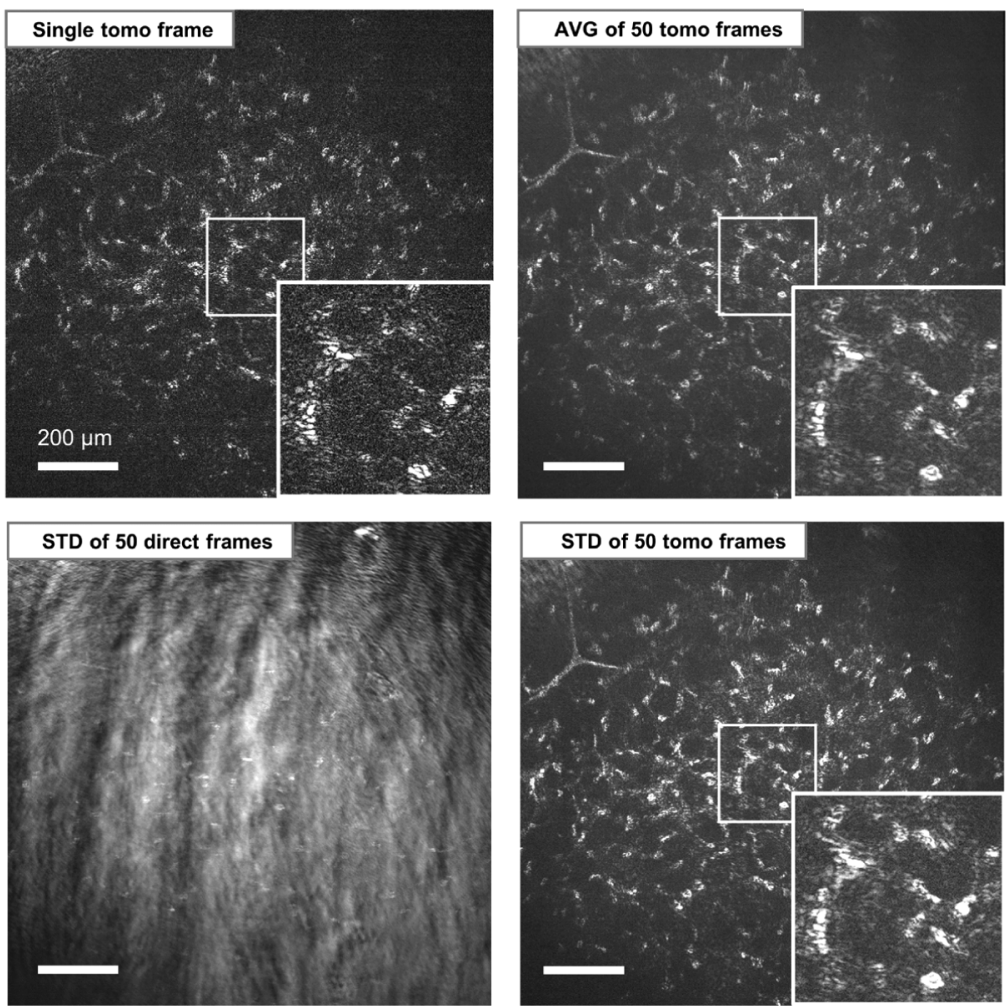

Fig. 11. Comparison of TD-FF-OCT images from in vivo human corneal stroma reconstructed from multiple frames via averaging or standard deviation. All scale bars are 200 $\mu \mathrm{m}$.

\subsection{Influence of lateral motion}

We also explored the influence of lateral motion on TD-FF-OCT images. The two fastest sources of lateral motion are saccades, rotating the eye at about 14\% [36], and the movement of the tear film, spreading after the blink at about $4 \mathrm{~mm} / \mathrm{s}$ [29].

First, we checked, if the saccadic lateral movement can shift the corneal axial position (leading to an axial optical phase shift). Assuming that the center of the corneal curvature does not match with the rotation axis of the eyeball, we theoretically estimated the negligible axial corneal shift on a millisecond timescale $(\sim 0.3 \mathrm{~nm} / \mathrm{ms})$. Although, saccades do not produce significant axial shift, they substantially shift the cornea laterally $(\sim 3 \mu \mathrm{m} / \mathrm{ms})$. This shift is larger than the typical lateral resolution in our TD-FF-OCT microscope, thus it affects the tomographic images. More precisely, when the shift between the consecutive camera images is large, the incoherent light outside of the coherence volume is not completely removed during the consecutive image subtraction (see Eq. (1)). As most of the incoherent light originates from the air - tear film interface, the tomographic image reveals artefacts of structures from the corneal surface (Fig. 13). Saccadic motion shifts the structures within the coherence volume as well, which also suppresses the tomographic signal.

Unfortunately, the possibilities of lateral movement correction for signal restoration are limited. More precisely, in order to correct for lateral movements affecting the tomographic signal, the image registration should be applied to the direct images from the camera (frames directly captured by the camera without post-processing) and not the tomographic frames. The direct camera frames contain both coherent and incoherent light, therefore they have too low contrast for 

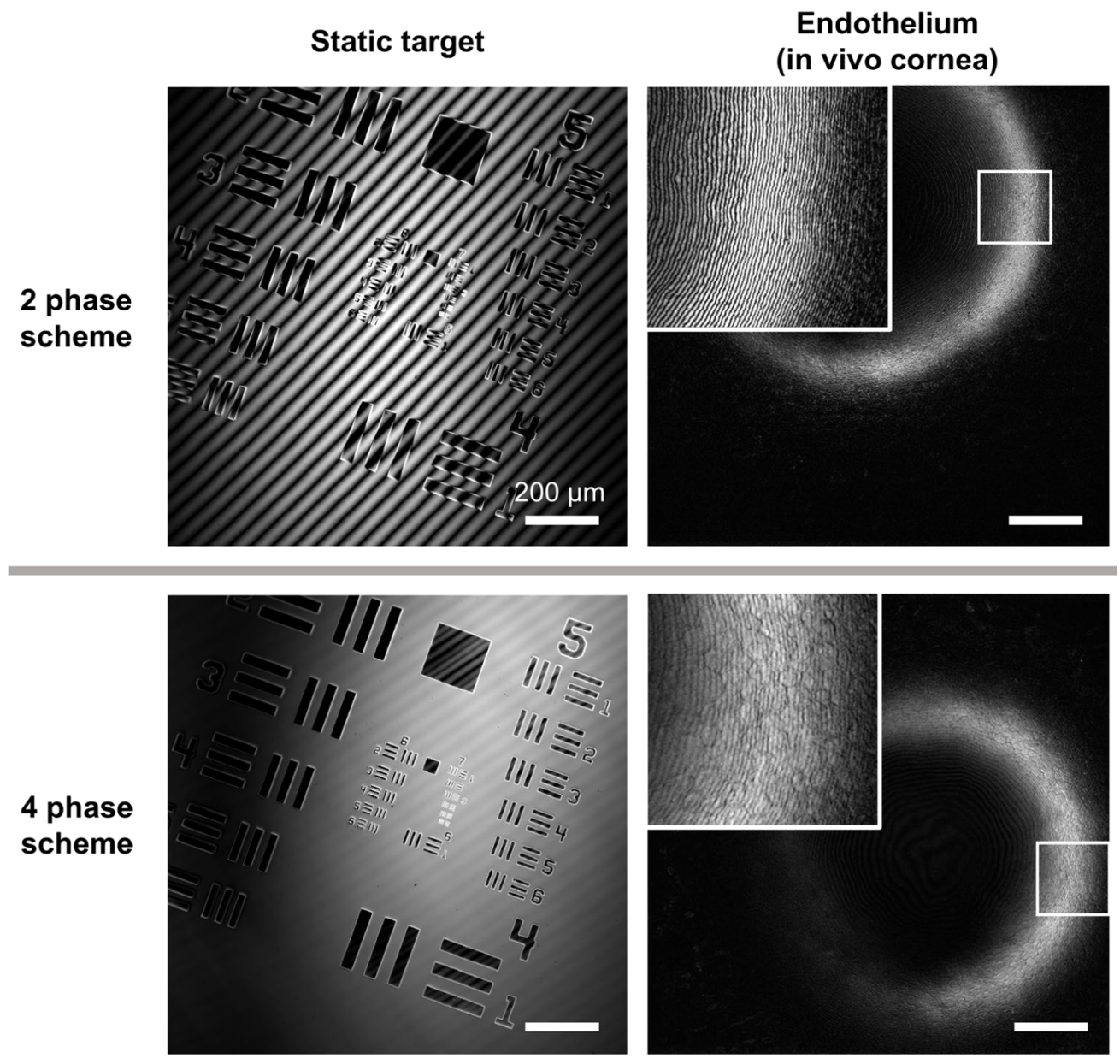

Fig. 12. Comparison of two- and four-phase modulation schemes. In vivo corneal images were acquired without piezo motor. The total exposure times were $3.5 \mathrm{~ms}$ for 2 phase and $7 \mathrm{~ms}$ for 4 phase images. Target images were acquired using the piezo modulation. The four-phase scheme suppresses the fringe artifacts that are typically present in uniform layers, such as the endothelium. All scale bars are $200 \mu \mathrm{m}$. 

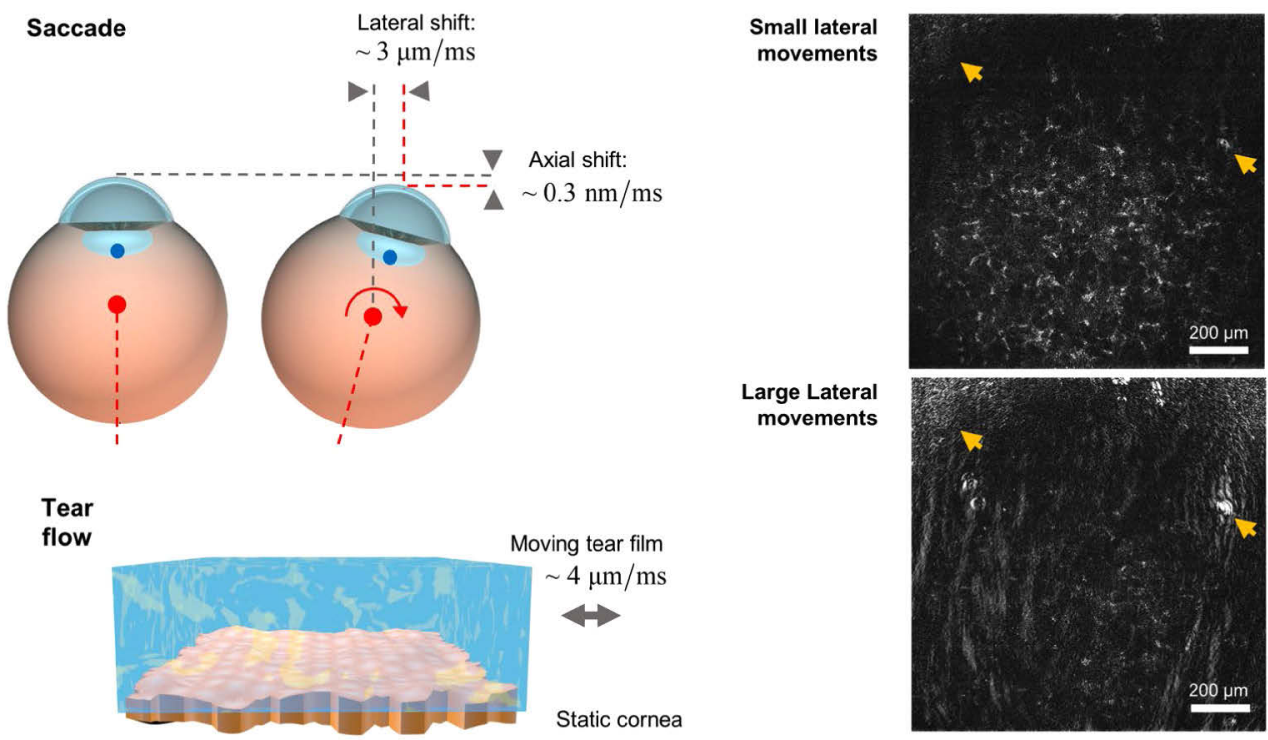

Fig. 13. Sources of lateral motion and their effects on TD-FF-OCT signal. On the millisecond timescale saccades, produce small axial and large lateral shifts. The large lateral shifts create the incoherent artefacts, more precisely the defocused view of the corneal surface (yellow arrows), and reduce the tomographic signal. Both images on the right were acquired from the same plane in human cornea in vivo. Tear flow produces similar surface artefacts during one second following the blink, while the tear velocity is high.

reliable registration. Moreover, most of the light in the frame comes from the surface reflection and not the tomographic layer of interest that should be aligned.

Following the blink, the tear film moves rapidly $(\sim 4 \mu \mathrm{m} / \mathrm{ms})$, but independently from the underlying static cornea. Therefore, it produces the same incoherent surface artifacts as saccades, but does not significantly reduce the tomographic signal. One second after the blink the tear film velocity reduces below $\sim 1 \mu \mathrm{m} / \mathrm{ms}$ [29] and almost all lateral artifacts disappear.

\subsection{Motion and blood flow imaging}

Lastly, we explored the effect of motion on blood flow imaging. Images with the lateral flow across the FOV present the highest interest for TD-FF-OCT as they allow quantitative measurements $[28,29]$. The lateral flow does not contribute to axial phase shifting, therefore the same analysis as the above applies. However, it is interesting to note that angiography images can be obtained even with the static eye and static piezo motor. More precisely, the dynamics of flowing blood cells $(\sim 1 \mu \mathrm{m} / \mathrm{ms})$ lead to fluctuations of brightness from one image to the next in a recorded sequence. When the difference of consecutive image is computed, the background is removed and the blood flow is highlighted (Fig. 14). This imaging approach reveals all the vessels within the depth-of-focus, meaning that it has poorer optical sectioning than the coherence gate width, but it works even if the reference arm is blocked as in conventional microscopy. 

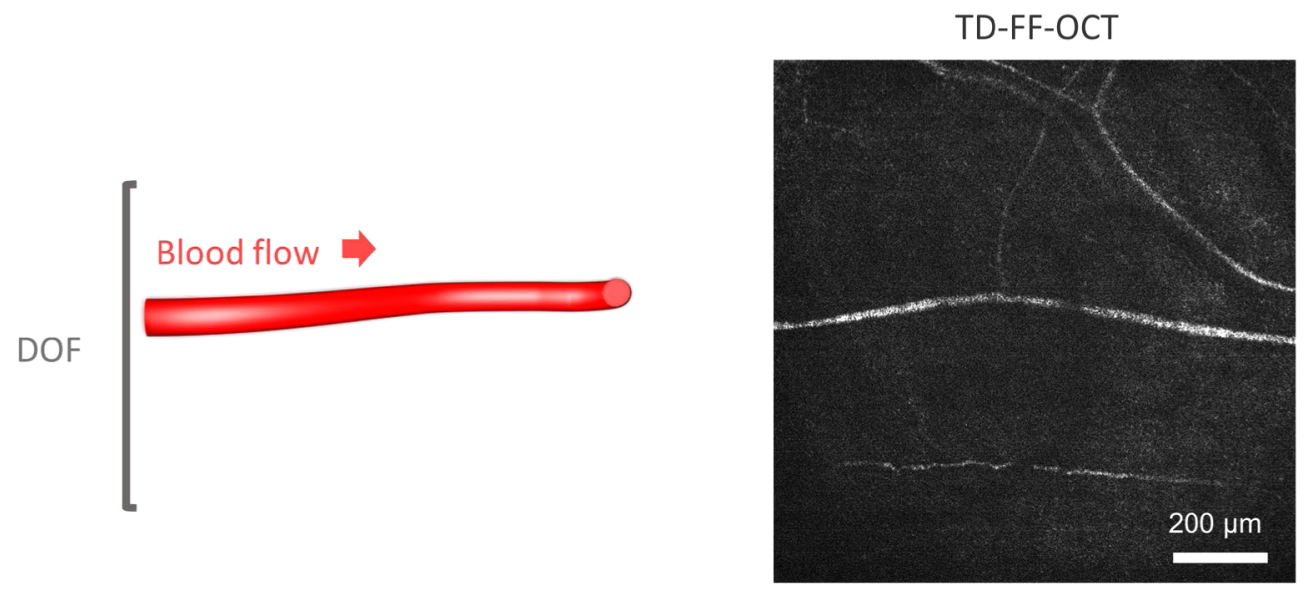

Fig. 14. Blood flow and TD-FF-OCT. Blood flow creates fluctuations in brightness from one image to the next one in a sequence. As a result it is visible even if the vessel lies outside of the coherence plane (or equivalently, when the reference arm is blocked). TD-FF-OCT reveals all the vessels within the depth-of-focus (DOF).

\section{Discussion and summary}

In this article we have studied the axial movements of the human eye at different time scales. The acquired knowledge about the distribution of axial eye movements can potentially be useful for improving existing axial eye tracking and correction methods, such as described in [29,33,37-43]. During exposure times below $0.1 \mathrm{~ms}$, the eye shifts by less than $100 \mathrm{~nm}$, equivalent to an optical phase shift of $\pi / 2$ for an $850 \mathrm{~nm}$ light wavelength. This confirms immunity of fast microsecond-exposure instruments to phase washout. During more than $1 \mathrm{~ms}$ the eye can move axially by more than $1 \mu \mathrm{m}$, leading to large optical phase shifts of $\pi, 2 \pi, 3 \pi$, etc. While playing a negative role for many ophthalmic instruments, these large phase shifts can be useful in time-domain methods for path-length modulation within the interferometer, substituting the commonly used piezo motor. However, it should be noted that modulation with eye movements only (without piezo) limits the maximal achievable signal to $70 \%$ (compared to the theoretical $100 \%$ maximum with static sample and piezo modulation). This is due to the continuous linear nature of eye motion on a millisecond timescale. By adding the discontinuous step-like piezo modulation, $100 \%$ of the signal can be recovered.

The proportion of images having sufficient tomographic signal depends on the exposure time and amplitude of the piezo modulation. When the exposure time is relatively large above 1 $\mathrm{ms}$, the phase modulation is dominated by the ocular movements and the probability of getting tomographic images is not affected by the piezo modulation. For example, the proportion is fixed at $50 \%$ (every $2^{\text {nd }}$ image) for a $1 \mathrm{~ms}$ exposure. Below $1 \mathrm{~ms}$, the speed of eye movements gradually becomes insufficient to provide the necessary $\pi$ phase shift between the frames. In this case the phase modulation with piezo motor becomes essential for capturing the tomographic signals.

Experimentally, tomographic images of the in vivo human cornea could be revealed with the TD-FF-OCT instrument at $3.5 \mathrm{~ms}$ ( $275 \mathrm{fps}), 10 \mathrm{~ms}$ (100 fps) and $20 \mathrm{~ms}$ (50 fps) exposure times (two camera frames) using only the phase shift, induced by the eye movements without piezo motor. This opens a path to a simplified ophthalmic TD-FF-OCT device, which uses a cheaper low-speed camera (that nonetheless still has high full-well capacity) and dispenses with the piezo motor-camera synchronization chain. It should be noted that: 1) TD-FF-OCT needs to acquire at least two frames on the camera (at $100 \mathrm{fps}$ ) to reconstruct the tomographic image (at $50 \mathrm{fps}$ ), 2) the lateral eye movements set the lower bound for the feasible lowest camera speed. 
A stack of multiple tomographic images originating from the same coherence volume can be processed to obtain a high SNR image even, if each image is being acquired with a different phase determined by the eye movements.

Finally, by using the natural eye movements it is possible to acquire not only two-phase, but also four-phase tomographic images. The latter have a benefit in that they suppress the unwanted interference fringe artifacts that are particularly visible on deterministic interfaces. The probability of getting the ideal phase shifts between the four consecutive images is negligibly low, nevertheless perfect matching is not required and visibility of interference fringe artifacts can be considerably reduced. This enables imaging of corneal endothelial cell mosaic using a single tomographic frame.

Funding. Centre National de la Recherche Scientifique (pre-maturation grant); Region Ile-De-France fund SESAME (EX047007); Association Instituts Carnot (x16-CARN 0029-01); Agence Nationale de la Recherche (ANR-18-IAHU0001); Agence Nationale de la Recherche (ANR-10-IDEX-0001-02 PSL); European Research Council (957546); European Research Council (610110).

Acknowledgments. We would like to acknowledge the advisory support of the Quinze-Vingts National Ophthalmology Hospital.

Disclosures. VM: (P), PX: (P), MF: (P), ACB: (P).

Data Availability. Data underlying the results presented in this paper are available in Dataset 1, Ref. [34].

\section{References}

1. R. de Kinkelder, J. Kalkman, D. J. Faber, O. Schraa, P. H. B. Kok, F. D. Verbraak, and T. G. van Leeuwen, "Heartbeat-induced axial motion artifacts in optical coherence tomography measurements of the retina," Invest. Ophthalmol. Vis. Sci. 52(6), 3908 (2011).

2. M. Hedley and H. Yan, "Motion artifact suppression: a review of post-processing techniques," Magn. Reson. Imaging 10(4), 627-635 (1992).

3. D. Huang, E. Swanson, C. Lin, J. Schuman, W. Stinson, W. Chang, M. Hee, T. Flotte, K. Gregory, and C. Puliafito, et al., "Optical coherence tomography," Science 254(5035), 1178-1181 (1991).

4. Wolfgang Drexler and James G. Fujimoto, Optical Coherence Tomography: Technology and Applications, 2 (Springer International Publishing, 2015).

5. S. H. Yun, G. J. Tearney, J. F. de Boer, and B. E. Bouma, "Motion artifacts in optical coherence tomography with frequency-domain ranging," Opt. Express 12(13), 2977 (2004).

6. B. J. Vakoc, S. H. Yun, J. F. de Boer, G. J. Tearney, and B. E. Bouma, "Phase-resolved optical frequency domain imaging," Opt. Express 13(14), 5483 (2005).

7. S. H. Yun, G. J. Tearney, J. F. de Boer, and B. E. Bouma, "Pulsed-source and swept-source spectral-domain optical coherence tomography with reduced motion artifacts," Opt. Express 12(23), 5614 (2004).

8. L. An, P. Li, T. T. Shen, and R. Wang, "High speed spectral domain optical coherence tomography for retinal imaging at 500,000 A-lines per second," Biomed. Opt. Express 2(10), 2770 (2011).

9. B. Tan, Z. Hosseinaee, L. Han, O. Kralj, L. Sorbara, and K. Bizheva, " $250 \mathrm{kHz}, 15 \mu \mathrm{m}$ resolution SD-OCT for in-vivo cellular imaging of the human cornea," Biomed. Opt. Express 9(12), 6569 (2018).

10. S. Yun, G. Tearney, J. de Boer, N. Iftimia, and B. Bouma, "High-speed optical frequency-domain imaging," Opt. Express 11(22), 2953 (2003).

11. I. Grulkowski, J. J. Liu, B. Potsaid, V. Jayaraman, C. D. Lu, J. Jiang, A. E. Cable, J. S. Duker, and J. G. Fujimoto, "Retinal, anterior segment and full eye imaging using ultrahigh speed swept source OCT with vertical-cavity surface emitting lasers," Biomed. Opt. Express 3(11), 2733 (2012).

12. H. C. Hendargo, R. P. McNabb, A.-H. Dhalla, N. Shepherd, and J. A. Izatt, "Doppler velocity detection limitations in spectrometer-based versus swept-source optical coherence tomography," Biomed. Opt. Express 2(8), 2175 (2011).

13. T. Klein, W. Wieser, L. Reznicek, A. Neubauer, A. Kampik, and R. Huber, "Multi-MHz retinal OCT," Biomed. Opt. Express 4(10), 1890 (2013).

14. T. Huo, C. Wang, X. Zhang, T. Chen, W. Liao, W. Zhang, S. Ai, J.-C. Hsieh, and P. Xue, "Ultrahigh-speed optical coherence tomography utilizing all-optical $40 \mathrm{MHz}$ swept-source," J. Biomed. Opt. 20(3), 030503 (2015).

15. A. F. Zuluaga and R. Richards-Kortum, "Spatially resolved spectral interferometry for determination of subsurface structure," Opt. Lett. 24(8), 519 (1999).

16. B. Považay, A. Unterhuber, B. Hermann, H. Sattmann, H. Arthaber, and W. Drexler, "Full-field time-encoded frequency-domain optical coherence tomography," Opt. Express 14(17), 7661 (2006).

17. Z. Hosseiaee, B. Tan, K. Bizheva, and L. Han, "High resolution line-field SD-OCT with $2.5 \mathrm{kHz}$ frame rate for cellular resolution imaging of biological tissue," in Optical Coherence Tomography and Coherence Domain Optical Methods in Biomedicine XXIII, J. A. Izatt and J. G. Fujimoto, eds. (SPIE, 2019), p. 104.

18. L. Ginner, A. Kumar, D. Fechtig, L. M. Wurster, M. Salas, M. Pircher, and R. A. Leitgeb, "Noniterative digital aberration correction for cellular resolution retinal optical coherence tomography in vivo," Optica 4(8), 924 (2017). 
19. D. Hillmann, H. Spahr, C. Hain, H. Sudkamp, G. Franke, C. Pfäffle, C. Winter, and G. Hüttmann, "Aberration-free volumetric high-speed imaging of in vivo retina," Sci. Rep. 6(1), 35209 (2016).

20. E. Auksorius, D. Borycki, P. Stremplewski, K. Liżewski, S. Tomczewski, P. Niedźwiedziuk, B. L. Sikorski, and M. Wojtkowski, "In vivo imaging of the human cornea with high-speed and high-resolution Fourier-domain full-field optical coherence tomography," Biomed. Opt. Express 11(5), 2849 (2020).

21. P. Stremplewski, E. Auksorius, P. Wnuk, Ł Kozoń, P. Garstecki, and M. Wojtkowski, "In vivo volumetric imaging by crosstalk-free full-field OCT," Optica 6(5), 608 (2019).

22. C. Pfäffle, H. Spahr, D. Hillmann, H. Sudkamp, G. Franke, P. Koch, and G. Hüttmann, "Reduction of frame rate in full-field swept-source optical coherence tomography by numerical motion correction [Invited]," Biomed. Opt. Express 8(3), 1499 (2017).

23. E. Beaurepaire, A. C. Boccara, M. Lebec, L. Blanchot, and H. Saint-Jalmes, "Full-field optical coherence microscopy," Opt. Lett. 23(4), 244 (1998).

24. G. Moneron, A. C. Boccara, and A. Dubois, "Stroboscopic ultrahigh-resolution full-field optical coherence tomography," Opt. Lett. 30(11), 1351 (2005).

25. K. Grieve, G. Moneron, A. Dubois, J.-F. L. Gargasson, and C. Boccara, "Ultrahigh resolution ex vivo ocular imaging using ultrashort acquisition time en face optical coherence tomography," J. Opt. A: Pure Appl. Opt. 7(8), 368-373 (2005).

26. H. Sudkamp, P. Koch, H. Spahr, D. Hillmann, G. Franke, M. Münst, F. Reinholz, R. Birngruber, and G. Hüttmann, "In-vivo retinal imaging with off-axis full-field time-domain optical coherence tomography," Opt. Lett. 41(21), 4987 (2016).

27. C. Apelian, F. Harms, O. Thouvenin, and A. C. Boccara, "Dynamic full field optical coherence tomography: subcellular metabolic contrast revealed in tissues by interferometric signals temporal analysis," Biomed. Opt. Express 7(4), 1511 (2016).

28. J. Scholler, V. Mazlin, O. Thouvenin, K. Groux, P. Xiao, J.-A. Sahel, M. Fink, C. Boccara, and K. Grieve, "Probing dynamic processes in the eye at multiple spatial and temporal scales with multimodal full field OCT," Biomed. Opt. Express 10(2), 731 (2019).

29. V. Mazlin, P. Xiao, J. Scholler, K. Irsch, K. Grieve, M. Fink, and A. C. Boccara, "Real-time non-contact cellular imaging and angiography of human cornea and limbus with common-path full-field/SD OCT," Nat. Commun. 11(1), $1868(2020)$.

30. V. Mazlin, P. Xiao, E. Dalimier, K. Grieve, K. Irsch, J.-A. Sahel, M. Fink, and A. C. Boccara, "In vivo high resolution human corneal imaging using full-field optical coherence tomography," Biomed. Opt. Express 9(2), 557 (2018).

31. V. Mazlin, K. Irsch, K. Irsch, M. Paques, J.-A. Sahel, J.-A. Sahel, J.-A. Sahel, M. Fink, and C. A. Boccara, "“'Curved-field optical coherence tomography: large-field imaging of human corneal cells and nerves," Optica 7(8), $872-880(2020)$

32. P. Xiao, V. Mazlin, K. Grieve, J.-A. Sahel, M. Fink, and A. C. Boccara, "In vivo high-resolution human retinal imaging with wavefront-correctionless full-field OCT," Optica 5(4), 409 (2018).

33. P. Mecê, J. Scholler, K. Groux, and C. Boccara, "High-resolution in-vivo human retinal imaging using full-field OCT with optical stabilization of axial motion," Biomed. Opt. Express 11(1), 492 (2020).

34. V. Mazlin, "Axial movements of healthy in vivo human eye," figshare (2021), https://doi.org/10.6084/m9.figshare.16752001.

35. A. Dubois, Handbook of Full-Field Optical Coherence Microscopy: Technology and Applications (Pan Stanford Publishing, 2016).

36. C. K. Sheehy, Q. Yang, D. W. Arathorn, P. Tiruveedhula, J. F. de Boer, and A. Roorda, "High-speed, image-based eye tracking with a scanning laser ophthalmoscope," Biomed. Opt. Express 3(10), 2611 (2012).

37. M. Pircher, B. Baumann, E. Götzinger, H. Sattmann, and C. K. Hitzenberger, "Simultaneous SLO/OCT imaging of the human retina with axial eye motion correction," Opt. Express 15(25), 16922 (2007).

38. M. Pircher, E. Götzinger, H. Sattmann, R. A. Leitgeb, and C. K. Hitzenberger, "In vivo investigation of human cone photoreceptors with SLO/OCT in combination with 3D motion correction on a cellular level," Opt. Express 18(13), 13935 (2010).

39. K. V. Vienola, B. Braaf, C. K. Sheehy, Q. Yang, P. Tiruveedhula, D. W. Arathorn, J. F. de Boer, and A. Roorda, "Real-time eye motion compensation for OCT imaging with tracking SLO," Biomed. Opt. Express 3(11), 2950 (2012).

40. Y. Chen, Y.-J. Hong, S. Makita, and Y. Yasuno, "Three-dimensional eye motion correction by Lissajous scan optical coherence tomography," Biomed. Opt. Express 8(3), 1783 (2017).

41. M. F. Kraus, B. Potsaid, M. A. Mayer, R. Bock, B. Baumann, J. J. Liu, J. Hornegger, and J. G. Fujimoto, "Motion correction in optical coherence tomography volumes on a per A-scan basis using orthogonal scan patterns," Biomed Opt. Express 3(6), 1182 (2012).

42. R. J. Zawadzki, A. R. Fuller, S. S. Choi, D. F. Wiley, B. Hamann, and J. S. Werner, "Correction of Motion Artifacts and Scanning Beam Distortions in 3D Ophthalmic Optical Coherence Tomography Imaging," in F. Manns, P. G. Soederberg, A. Ho, B. E. Stuck, and M. Belkin, eds. (2007), p. 642607.

43. M. Cua, S. Lee, D. Miao, M. J. Ju, P. J. Mackenzie, Y. Jian, and M. V. Sarunic, "Retinal optical coherence tomography at $1 \mu \mathrm{m}$ with dynamic focus control and axial motion tracking," J. Biomed. Opt 21(2), 026007 (2016). 